\title{
Hysteresis Analysis of Three Phase Voltage Source PWM Rectifier based on Feed Forward Decoupling
}

\author{
Cheng Wang ${ }^{1, a}$ \\ ${ }^{1}$ School of Electrical Engineering, Xi'an Aeronautical University, Xi'an, 710077 \\ ${ }^{a}$ email
}

Keywords: PWM Rectifier, the Total Harmonic Distortion, Hysteresis, Feed Forward Decoupling, Matlab/Simulink

\begin{abstract}
In power electronic converter device, using the ordinary thyristor phased rectifier, to inject a large number of harmonics and reactive power grid, power grid pollution; With full control of PWM rectifier device, which can realize the grid side current according to the sine law of change, and run in unit power factor, reduce the pollution to the grid. By establishing the model of three-phase voltage source PWM rectifier, this paper analyzes the working principle of feed forward decoupling control strategy, structures, based on Matlab/Simulink simulation model, the running results show that the system works well, to meet the design requirements.
\end{abstract}

\section{Introduction}

In the power system, the harmonic distortion will cause many problems, such as by the control device diode, semi-control device thyristor form of the traditional rectifier, due to the power grid into a large number of harmonic components, resulting in reduced power factor seriously affected the power grid Of the safe operation, and cause other electronic equipment does not work [1]. In order to solve this problem, PWM rectifier begins to develop continuously. In this paper, by analyzing the three-phase voltage-type PWM rectifier model, a hysteresis current control method is proposed to reduce the harmonics, and the grid current is changed sinusoidally to meet the unit power factor of PWM rectifier. By running the simulation model and analyzing the waveform parameters, we achieve the design requirements.

\section{Three-Phase PWM Rectifier Model}

Three-phase voltage-type PWM rectifier main circuit from the full control device

The basic circuit shown in Fig. 1, mainly by the AC circuit, power switching circuit, DC circuit and PWM control module circuit. AC circuit includes the AC voltage source and the power side inductance [2]; DC circuit includes the load resistance and DC side capacitor, power switch circuit consists of six full-controlled IGBT composition. DC-side capacitor for the PWM rectifier output stability of the DC voltage to provide a guarantee, and can filter out the DC voltage pulsation components. Since the n-th harmonics contained in the DC-side busbar voltage are modulated, higher harmonics are generated in the line-side current, and therefore, the capacitor has the effect of reducing current harmonics. If the capacitance value is too large, the harmonic content of the DC voltage can be reduced and the disturbance capacity of the load can be improved. However, if the capacitance value is too large, the PWM rectifier volume will increase and affect the dynamic performance of the system. Capacitance value is too small, can not guarantee the DC side output voltage stability. 


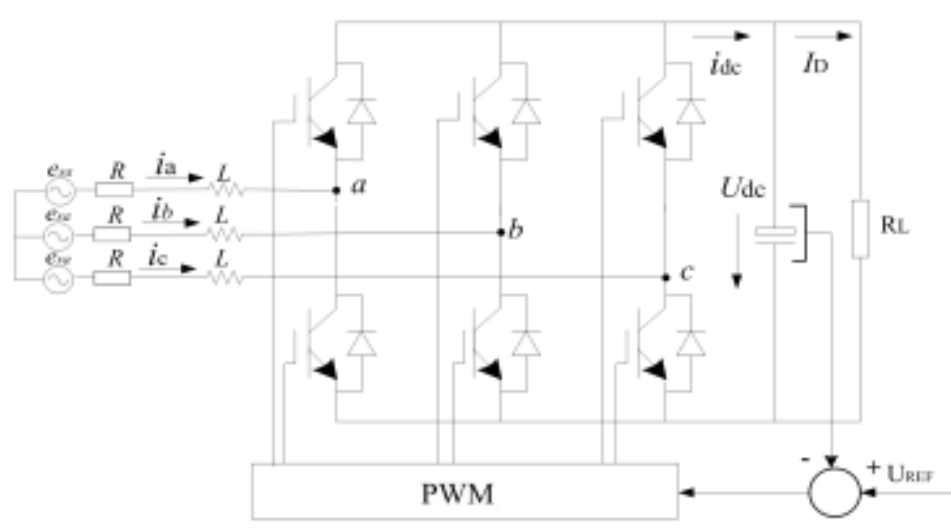

Fig. 1 Three-phase voltage type PWM rectifier

PWM control module circuit according to different control methods can be composed of the corresponding system [3]. PWM rectifiers to ensure that the output voltage is stable, the use of feedback has closed-loop control. To achieve the goal, the output side of the voltage across the capacitor $\mathrm{C}$ and a given reference voltage difference, the difference after the signal is generated, after the PWM control module operation processing, control PWM rectifier 6 off, control network side current, the current Same as the grid voltage and sinusoidal.

When the DC side current is positive (ie, rectifier mode), the output side of the capacitor C discharge, the resulting differential signal requires PWM control module to extract more power from the power side, the control module generates the appropriate signal control PWM rectifier switch In this mode, more current flows from the AC side to the DC side, and the capacitor voltage is restored [4]. When the current is negative (ie, inverter mode), the process is reversed. Using PWM control method, not only can realize the active power and reactive power control, and can achieve the AC side of the current to maintain sine, greatly reducing the harmonic pollution on the grid.

Fig. 1 shows, PWM rectifier using three-phase sinusoidal AC power supply, the main circuit from the six anti-parallel diodes with fully-controlled switching tube composed of these switches is mainly used to implement PWM control signal can be realized two-way flow of energy. And inductance in series with the power supply directly affect the working state of the rectifier, inductance value is too small will increase the current harmonic; if the increase in inductance value, although the current harmonics can be reduced, but will slow down the system response, in the circuit Need to be calculated by the specific parameters.

PWM rectifier with output DC voltage stability, energy can be two-way flow, current harmonic distortion is small, etc., have been widely used.

\section{Three-phase PWM Rectifier Model Analysis}

Three-phase voltage-type PWM rectifier power supply voltage and current expression (1)

$$
\left\{\begin{array}{l}
e_{a}(t)=E \sin (\omega t) \\
e_{b}(t)=E \sin \left(\omega t-\frac{2}{3} \pi\right) \\
e_{c}(t)=E \sin \left(\omega t-\frac{4}{3} \pi\right)
\end{array}\right.
$$

Where, $e_{a}, e_{b}, e_{c}$ three-phase power supply voltage, $E$ respectively, for the three-phase power supply voltage amplitude, $\omega$ angular frequency $\omega=2 \pi f$,.

Therefore, in the three-phase static coordinate system, can be obtained three-phase PWM rectifier mathematical model, such as (2) [5]: 


$$
\left\{\begin{array}{l}
L \frac{d i_{a}}{d t}+R i_{a}=u_{a}-e_{a} \\
L \frac{d i_{b}}{d t}+R i_{b}=u_{b}-e_{b} \\
L \frac{d i_{c}}{d t}+R i_{c}=u_{c}-e_{c}
\end{array}\right.
$$

In the formula (2), $\mathrm{L}$ is the inductance of the $\mathrm{AC}$ side, $\mathrm{R}$ is the equivalent resistance of the wire, $i_{a} 、 i_{b} 、 i_{c}$ is the output current of the three-phase power source, $u_{a} 、 u_{b}, u_{c}$ is the AC side voltage of the PWM rectifier.

Three-phase sinusoidal AC power supply voltage for the balance system, type (3) is established,

$$
\left\{\begin{array}{l}
e_{a}+e_{b}+e_{c}=0 \\
i_{a}+i_{b}+i_{c}=0
\end{array}\right.
$$

In order to simplify the design of the system and realize the independent control of the controlled volume, it is necessary to use the coordinate transformation to transform the three-phase static coordinate system into three-phase static coordinate system Of the exchange of two-phase synchronous rotating coordinate system to transform the amount of DC [6]. Equation (4) is the transformation of the variable into three-phase stationary coordinate system, (5) is the two-phase stationary coordinate system transformed into two-phase synchronous rotating coordinate system [7]

$$
\begin{aligned}
& {\left[\begin{array}{l}
u_{a} \\
u_{b} \\
u_{c}
\end{array}\right]=\sqrt{\frac{2}{3}}\left[\begin{array}{cc}
1 & 0 \\
-\frac{1}{2} & -\frac{\sqrt{3}}{2} \\
-\frac{1}{2} & \frac{\sqrt{3}}{2}
\end{array}\right]\left[\begin{array}{l}
u_{\alpha} \\
u_{\beta}
\end{array}\right]} \\
& {\left[\begin{array}{l}
u_{\alpha} \\
u_{\beta}
\end{array}\right]=\left[\begin{array}{cc}
\cos \omega t & \sin \omega t \\
-\sin \omega t & \cos \omega t
\end{array}\right]\left[\begin{array}{l}
u_{d} \\
u_{q}
\end{array}\right]}
\end{aligned}
$$

In the equation (4), $u_{\alpha} 、 u_{\beta}$ the voltage variable in the two-phase stationary coordinate system $u_{d} 、 u_{q}$ is the voltage variable in the two-phase synchronous rotating coordinate system. Using the above transformation formula, we can obtain the expression (6)

$$
\begin{aligned}
& \left\{\begin{array}{l}
L \frac{d i_{\alpha}}{d t}+R i_{\alpha}=u_{\alpha}-e_{\alpha} \\
L \frac{d i_{\beta}}{d t}+R i_{\beta}=u_{\beta}-e_{\beta}
\end{array}\right. \\
& \left\{\begin{array}{l}
L \frac{d i_{d}}{d t}+R i_{d}-\omega L i_{q}=u_{d}-e_{d} \\
L \frac{d i_{q}}{d t}+R i_{q}+\omega L i_{d}=u_{q}-e_{q}
\end{array}\right.
\end{aligned}
$$

\section{Three-phase PWM Rectifier}

From the above analysis, it can be seen that the $\mathrm{d}$ and $\mathrm{q}$ axis variables of the PWM rectifier in the two-phase synchronous rotating coordinate system are coupled with each other and the design system is difficult to realize. Therefore, the mathematical model is decoupled. Transform (7) can be obtained formula (8): 


$$
\left\{\begin{array}{l}
u_{d}=e_{d}-\omega L i_{q}-R i_{d}-L \frac{d i_{d}}{d t} \\
u_{q}=e_{d}+\omega L i_{d}-R i_{q}-L \frac{d i_{q}}{d t}
\end{array}\right.
$$

To improve the current waveform, the PWM rectifier in the unity power factor under the current feedback loop based on the hysteresis control method, when the actual value and the difference between the value of more than a specified range, will produce a control PWM rectifier Power switch signal [9]. The output value is adjusted by PI and is obtained by adjusting the actual value of the current and the reference value. (9)

$$
\left\{\begin{array}{l}
R i_{d}+L \frac{d i_{d}}{d t}=\left(k_{p}+\frac{k_{i}}{s}\right)\left(i_{d}^{*}-i_{d}\right) \\
R i_{q}+L \frac{d i_{q}}{d t}=\left(k_{p}+\frac{k_{i}}{s}\right)\left(i_{q}^{*}-i_{q}\right)
\end{array}\right.
$$

(8) $k_{p}$ is substituted into (9), $k_{i}$ is the linear model of current decoupling can be obtained completely. The equation of state is shown in equation (10): () () () ()

$$
p\left[\begin{array}{l}
i_{d} \\
i_{q}
\end{array}\right]=\frac{1}{L}\left[\begin{array}{rc}
\left(k_{p}+\frac{k_{i}}{s}\right)-R & 0 \\
0 & \left(k_{p}+\frac{k_{i}}{S}\right)-R
\end{array}\right]\left[\begin{array}{l}
i_{d} \\
i_{q}
\end{array}\right]-\frac{1}{L}\left(k_{p}+\frac{k_{i}}{s}\right)\left[\begin{array}{l}
i_{d}^{*} \\
i_{q}^{*}
\end{array}\right]
$$

As can be seen from the above equation, the decoupling method can realize decoupling of the alternating current measured in the two-phase synchronous rotating coordinate system of the PWM rectifier, and can independently control the active current and the reactive current to make the design of the current loop easier.

Control system structure shown in Fig. (2):c

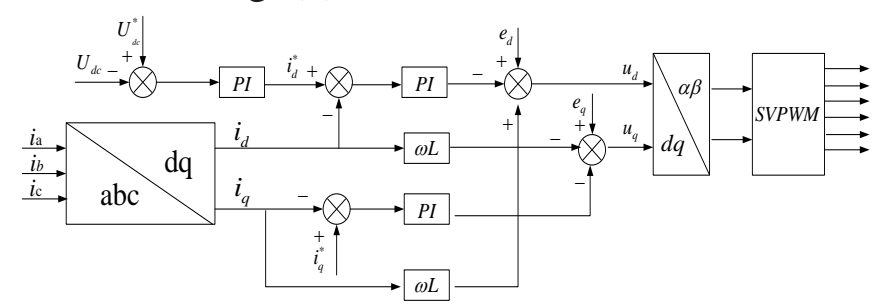

Fig. 2 three-phase voltage-type PWM rectifier control system diagram

\section{Simulation Results Analysis}

According to the above three-phase PWM rectifier system block diagram, using Matlab / Simulink to build simulation model, the model structure shown in Fig. (3), the main parameters of the parameters shown in Table 1:

\begin{tabular}{|c|c|}
\hline Parameter & Set value \\
\hline Three phase AC power supply phase voltage & $220 \mathrm{~V}$ \\
\hline Power frequency & $50 \mathrm{~Hz}$ \\
\hline AC side inductance & $4.3 \mathrm{mH}$ \\
\hline DC side capacitor & $2000 \mathrm{uF}$ \\
\hline DC side reference voltage & $660 \mathrm{~V}$ \\
\hline
\end{tabular}




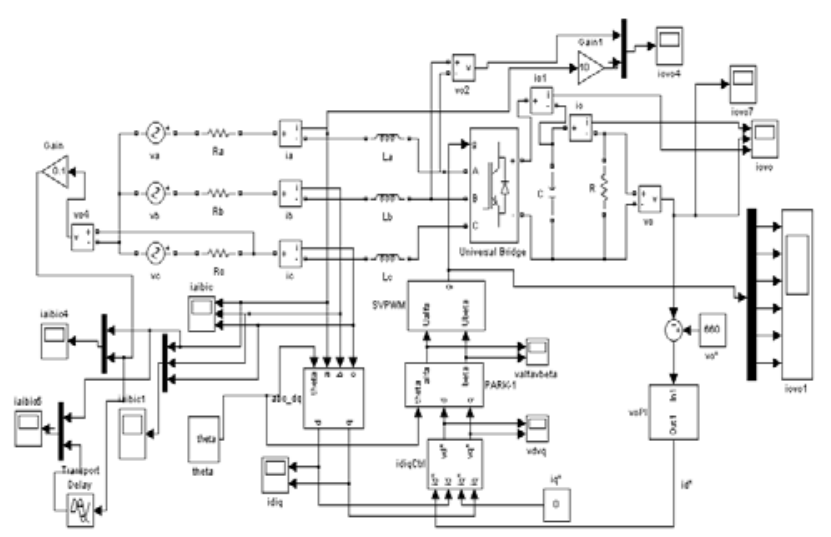

Fig. 2 Simulink simulation model

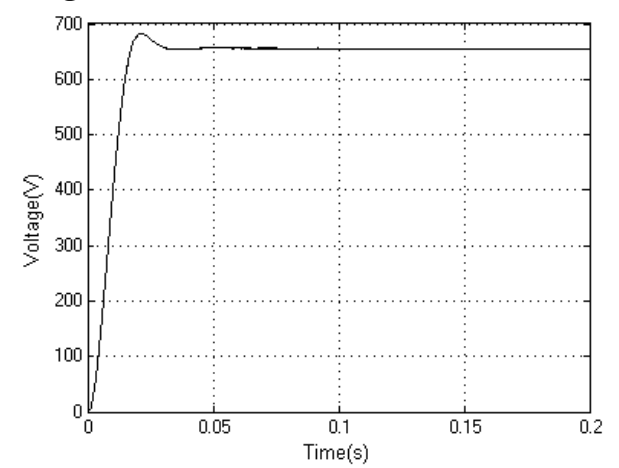

Fig. 3 DC side voltage waveform

It can be seen from Fig. 3: DC side output voltage after about 0.05 s to reach a stable state, the output value and set the same value.

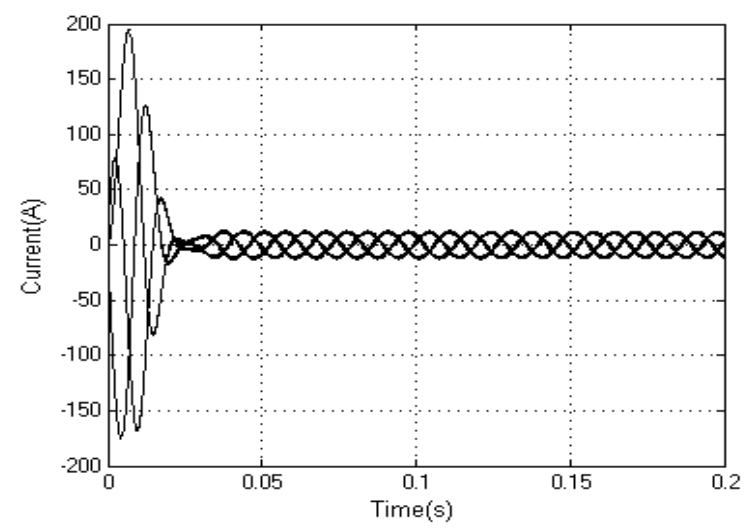

Fig. 4 power supply current waveform

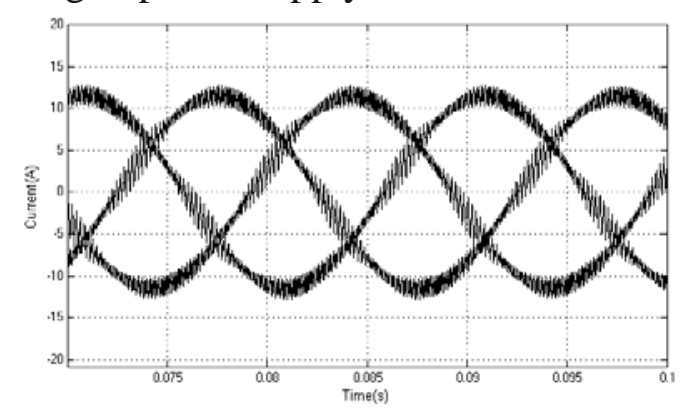

Fig. 5 shows the supply current detail waveform

Fig. 4 for the power supply current waveform, adjusted by $0.03 \mathrm{~s}$, the output reaches a stable value. Fig. 5 shows the power supply current stable waveform, can be seen from Fig. 5: the power supply current waveform according to sinusoidal law, phase and frequency and voltage the same, and the amplitude in the set range adjustment, consistent with the hysteresis control characteristics. Fig. 7, Fig. 8 are for the AC side of the phase voltage and line voltage waveform. 


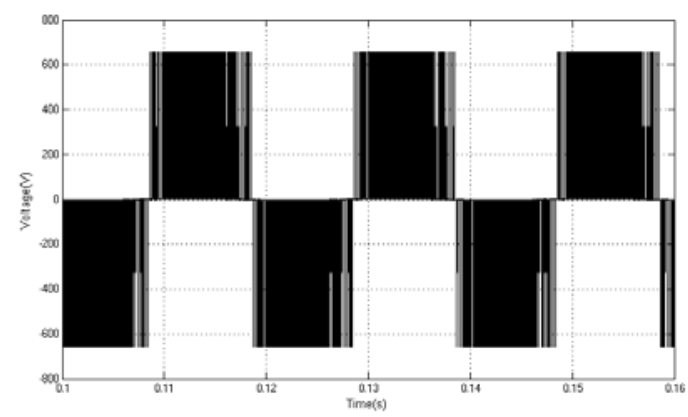

Fig. 6 phase voltage waveform

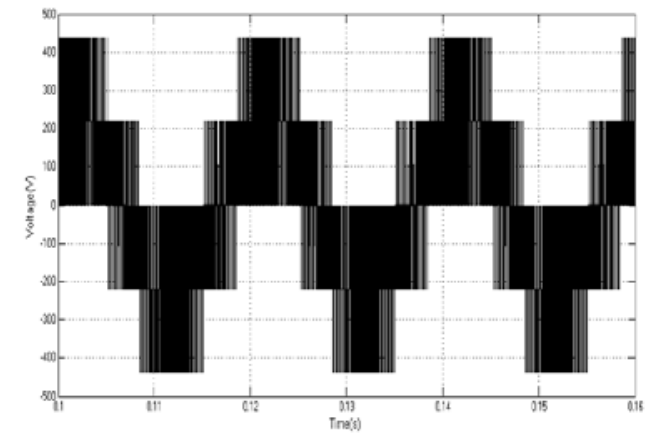

Fig. 7 Line voltage waveform
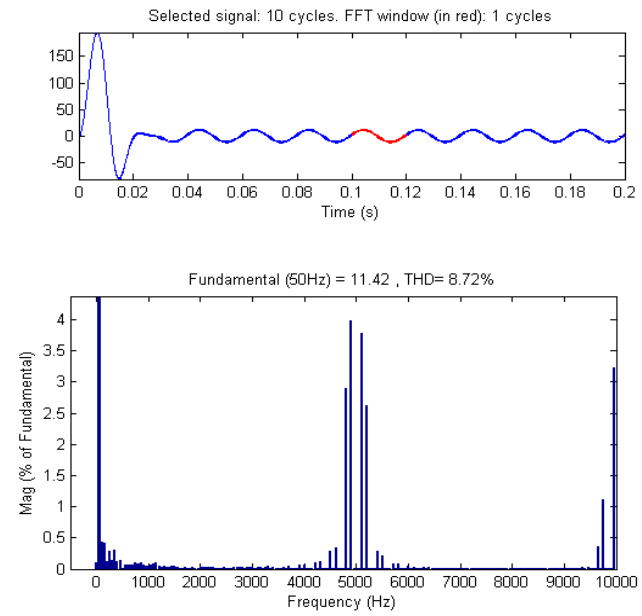

Fig. 8 Current harmonic analysis

Fig. 8 shows the power supply output current harmonic analysis, from Fig. 8 can be seen: THD value is small, to meet the design requirements.

\section{Conclusion}

In this paper, a novel PWM rectifier control model is proposed, which can improve the system performance by adjusting the parameters by using PI controller based on hysteresis current. Through the simulation analysis, the system output stability, with good power factor correction and sinusoidal current. So the system has a convenient control, good dynamic performance characteristics.

\section{References}

[1] Zhang Chongwei, Zhang Xing .PWM rectifier and its control [M]. Beijing: Mechanical Industry Press, 2012.

[2] Shen Chuanwen, Liu Wei. Research on three-phase rectifier based on feedforward decoupling [J]. Power Electronics Technology, 2006, 40 (2): 28-30.

[3] A. Semmah, A. Massoum, Patrice Wira Improvement of PWM Rectifier Performances Using 
Direct Power Control and Fuzzy Logic Theory, Australian Journal of Basic and Applied Sciences, 7 (4): 297-303, ISSN 1991-8178, .

[4] Zheng Zhongji. Three-phase voltage type PWM rectifier control strategy and application research [D]. Dalian University of Technology, 2011

[5] Himanshu Chaturvedi, Anil K. Chaudhary, C. Veeresh. Simulation of three-phase bridge rectifier using Matlab/Simulink for harmonic mitigation, International Journal of Modern Engineering Research (IJMER), Vol. 3, Issue 4, Jul-Aug. 2013 pp-2446-2450

[6] Hui Wang, Hui Qi, Study of Control Strategies for Voltage-Source PWM Rectifier Proceedings of the 2nd International Conference on Computer Science and Electronics Engineering (ICCSEE 2013) 1268-1271

[7] J. Lamterkati, M. Khafallah, L. Ouboubker, A New DPC for Three-phase PWM rectifier with unity power factor operation, International Journal of Advanced Research in Electrical, Electronics and Instrumentation Engineering Vol. 3, Issue 4, April 2014,8273-8285

[8] Zeng Xianjin, Li Xiaowei, Hu Likun, Ji Liwen, Lu Ziguang. Three-phase voltage type PWM converter control based on natural coordinates and power feedforward [J]. Power System Protection and Control, 2015, 43 (11): 13-20.

[9] Lu Baoliang, based on the three-phase voltage rectifier design [D]. Harbin: Harbin University of Science and Technology, 2007 